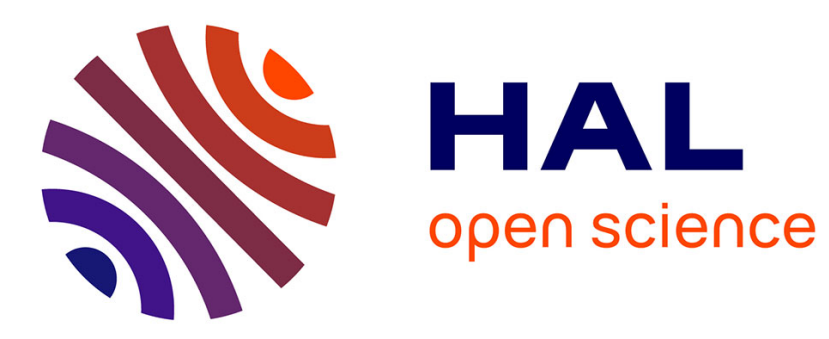

\title{
Application of numerical simulation in design and control of forming processes
}

\author{
J. Huétink, H. Mooi
}

\section{To cite this version:}

J. Huétink, H. Mooi. Application of numerical simulation in design and control of forming processes. Journal de Physique IV Proceedings, 1993, 03 (C7), pp.C7-1129-C7-1134. 10.1051/jp4:19937175 . jpa-00251806

\section{HAL Id: jpa-00251806 https://hal.science/jpa-00251806}

Submitted on 1 Jan 1993

HAL is a multi-disciplinary open access archive for the deposit and dissemination of scientific research documents, whether they are published or not. The documents may come from teaching and research institutions in France or abroad, or from public or private research centers.
L'archive ouverte pluridisciplinaire HAL, est destinée au dépôt et à la diffusion de documents scientifiques de niveau recherche, publiés ou non, émanant des établissements d'enseignement et de recherche français ou étrangers, des laboratoires publics ou privés. 


\title{
Application of numerical simulation in design and control of forming processes
}

\author{
J. HUÉTINK and H.G. MOOI
}

University of Twente, Department of Mechanical Engineering, P.O. Box 217, 7500 AE Enschede, The Netherlands

\begin{abstract}
A short overview is given of the development of the mixed Eulerian-Lagrangian formulation as a combination of the pure Eulerian and pure Lagrangian formulation. Emphasis is laid upon the formulation for forming processes, where the updating of material variables is important. Convection is added to the original Lagrangian method. Shortly, smoothing, the constitutive behaviour and a method to accelerate steady state calculations are treated. Two applications are shown: a rolling and an extrusion problem.
\end{abstract}

\section{INTRODUCTION}

Problems in solid mechanics are commonly formulated in material Lagrangian coordinates, because the difference in spatial position in the deformed and undeformed state is rather small. At the numerical simulation of more complicated forming processes one is faced with two sorts of nonlinearities, i.e.:

1) constitutive nonlinearities, caused by the complicated relation between stresses and strains of the materials used (after exceeding the elasticity limit), and

2) geometrical nonlinearities, encountered by large deformations (plastic deformations, postbuckling behaviour).

The total Lagrangian formulation proposed by Hibbitt, Marcal and Rice [1] is able to deal with the geometrical nonlinearities. In this method the equations are formulated relatively to the same reference state, which mostly coincides with the undeformed state. In elastic-plastic analysis the equations are rather complicated and, therefore, this method is not much applied. To be able to handle large inelastic deformations more suitably, McMeeking and Rice [2] introduced the updated Lagrangian method at which the reference state is updated after each increment and then coincides with the current state. An advantage of the Lagrangian description is the fact that deformation history dependent properties (strain hardening etc.) can be easily taken into account in a natural way, and the changing shape of the formed product can be followed. A restriction of the updated Lagrange method, though, is the limited deformation range because the element mesh may be completely distorted after a number of steps, disabling further calculation. This distortion can be avoided by mesh-rezoning or remeshing in updated Lagrange simulations (introduced by Gelten et al. [3]) in which history dependent properties are mapped from the old mesh to a new mesh. In some cases remeshing may not be efficient, for instance in a spatial area independent of the material flow (in regions with high rate of deformation or with changing contact between tool and nodal material points). In these cases remeshing is required too frequently. 
lation is very well-suited for many flow problems with fixed boundaries. Examples are the steady state continuous forming processes, such as wire drawing, extrusion and rolling. A strong disadvantage of the Eulerian formulation is that free surfaces and the interaction between flow and a structure usually can not be described. To solve these kind of problems, Hughes et al. [4] and Donea [5] introduced the Arbitrary Lagrangian-Eulerian (ALE) description in fluid dynamics. Furthermore, history or path dependent properties of the flow can not be treated properly as well. This is mainly caused by the fact that in Newtonian fluid problems no deformation history dependent material properties have to be taken into account and, thus, no particular attention has been paid to this aspect in the simulation of fluid problems. As an example, Liu et al. [6] developed an extension of the Eulerian method for path dependent materials.

To avoid the above mentioned problems with both the Lagrangian and Eulerian formulation, a procedure has been derived in which the location of the nodal points are not updated exactly according to the displacement of the material but are more or less disconnected in a way that the mesh is not too much distorted. Convective terms which are proportional to the (local) relative displacement increments are added in the Lagrangian update procedure for the history dependent material properties (see Huétink [7]). An updated Lagrange approach as well as an Eulerian approach can be regarded as special cases of the procedure, therefore, it is called the mixed Eulerian-Lagrangian formulation. In this way the advantages of the updated Lagrange method satisfying conditions for free or coupled surface movements as well as the updating of history dependent properties can be preserved, whereas internal mesh distortion can be avoided. The Arbitrary Eulerian Lagrangian formulation (ALE) has been developed for fluid dynamics simultaneously, though in this case as an extension of the Eulerian formulation (contrary to the method developed by Huétink).

\section{THE NUMERICAL METHOD}

First, a short description of the mixed Eulerian-Lagrangian formulation is given. In this formulation, the rate of change of the material associated quantities in each grid point has to be updated. Therefore, both the material rate of change (of an arbitrary magnitude $f$ ) $\mathrm{f}^{\mathrm{m}}$ as well as the rate of change in a grid point $\mathrm{f}^{\mathrm{g}}$ have to be examined. The magnitude $f$ represents the strains and the stresses. These rates read, respectively:

$$
\begin{aligned}
& \dot{f}^{m}=\left(\frac{d f}{d t}\right)^{m}=\frac{\partial f}{\partial t}+\dot{x}^{m} \cdot \underset{\nabla}{\vec{\nabla}} f \\
& \dot{f}^{\xi}=\left(\frac{d f}{d t}\right)^{g}=\frac{\partial f}{\partial t}+\dot{x}^{g} \cdot \underset{\nabla}{\vec{\nabla}} f
\end{aligned}
$$

where $\dot{x}^{m}$ and $\dot{x}^{8}$ represent the material and grid velocity, respectively. Strictly speaking, all the terms in eqs. (1) an (2) should be averaged during the time increment. Eliminating the first term on the right hand side of eqs.(1) and (2), and multiplying by a time increment $\Delta t$ yields a time incremental procedure:

$$
f\left(x^{g}+\Delta x^{g}, t+\Delta t\right)=f\left(x^{g}, t\right)+\Delta f^{m}+\left(\Delta x^{8}-\Delta x^{m}\right) \cdot \vec{\nabla} f
$$

This can be seen as a Taylor expansion of:

$$
f\left(x^{g}+\Delta x^{g}, t+\Delta t\right)=f\left(x^{g}+\Delta x^{g}-\Delta x^{m}, t\right)+\Delta f^{m}
$$

The last term in eq.(3) shows that if the new (updated) grid value has to be calculated in case of uncoupled material and grid points, convection has to be taken into account. This term hampers the 
calculation considerably, because the gradient of $f$ (representing stresses and strains) is needed. Since the stresses and strains are discontinuous over the element boundaries, a smoothing procedure has to be applied first (see Hinton [8] and Huétink [9]).

The smoothing comprises two stages: local smoothing (least square fit, mostly a lower order interpolation, at element level) and global smoothing (averaging of centroid element values of $f$ from the adjacent elements to the common nodal point). Using the smoothed field only for the determination of the gradient in (3) in order to update the state at integration points appeared to result into numerical instabilities depending on the size of the displacement increments. By applying the smoothed field completely, which means the use of eq.(4) instead of eq.(3), numerical instabilities can be avoided. Unfortunately, in this case too much smoothing due to numerical diffusion may occur. A solution has been found by weighing the two afore-mentioned formulations, by which the amount of global smoothing can be adjusted. The weight factor depends on the ratio of the displacement increment and the element size (Courant number) and is determined by a number of numerical experiments.

Furthermore, for two-dimensional simulations Huétink developed a method to diminish so-called crosswind diffusion. This method is an adaptation of the afore-mentioned smoothing procedure. The adaptation depends on the curvature of the field in the streamline direction. More details of this method as well as the smoothing procedure can be found in [9].

\section{INCREMENT MAGNIFICATION}

Some parts of steady state processes are characterized by the fact that the development of the distribution of certain magnitudes (almost) only takes place as a result of convection. The strain distribution of rolling or extrusion in the outlet zone can be taken as an example: no more plastic deformation occurs in that region and a mere shift of the strain distribution in the process direction has to be fulfilled. Unfortunately, this convection takes rather many increments, since the increments have to be small enough in order to satisfy the stability criterion. This stability criterion simply reads:

$$
v_{\text {dof }} \Delta t<C l_{\text {elt }} \quad \forall D O F s \text {, }
$$

where $1_{\text {elt }}$ represents the local element size and $C$ is a constant to account for the difference in sizes of adjacent elements.

In order to reach the steady state within fewer increments, Huétink and Meijers [10] proposed a method to accelerate the development of e.g. the strain distribution. This is accomplished by multiplying the local time increment by a magnification factor $\lambda_{m}\left(\lambda_{m}>1\right)$ so that, where possible, the convection is (almost) maximum. This maximum is first dictated by the stability criterion eq.(5) and is, furthermore, dependent on the degree of (in)stationarity of the underlying field. The result is that all increments in eq.(3) are multiplied by $\lambda_{\mathrm{m}}$. It should be emphasized that the resulting distribution is no longer physically correct, since the calculated state contains regions with different time histories.

For rolling and extrusion, the interesting magnitudes to be accelerated are the plastic strain, the stresses, and the temperature (the latter will not be treated here). They all need a different calculation method of their magnification factor $\lambda_{m}$. To start with, the plastic strain is rather insensitive for increment magnification. However, it suffers from two general negative properties of this method: first, increment magnification diminishes the accuracy because of truncation errors due to the shape of the source distribution. Secondly, application of too large magnification factors causes local overshoot. Although the overshoot disappears by convection after enough increments, it is preferred to prevent the overshoot by diminishing the magnification factor dependent on the relative magnitude of the material and convective increments (see [10]). If the material increment equals zero (outlet 
zone!), the maximum increment magnification can be applied.

The accelerated calculation of stresses is necessary in order to accomplish the residual stress distribution sooner. The stresses, though, are far more sensitive to increment magnification since they directly influence mechanical equilibrium, the base of the finite element method. So, apart from the measures analogous to those taken at plastic strain, some more evaluations of the stress field are done to check whether and how much increment magnification can be applied. If there are too many wiggles, for example, increment magnification is completely cancelled up to now (other solutions will be examined in the near future for this and other problems). Some results of this method are given at the end of this paper.

\section{MATERIAL BEHAVIOUR}

The material behaviour is described by the common elastic-plastic constitutive behaviour including thermal effects:

$$
\underline{\underline{\nabla}}=\frac{\dot{\rho}}{\rho} \underline{\underline{g}}+\underline{\mathbb{C}}: \underline{d}+\underline{\mathbb{K}} \dot{T}-\Phi^{t}
$$

where $\underset{\nabla}{\underline{\sigma}}$ is the Jaumann rate of the Cauchy stress tensor $\underline{\sigma}, \rho$ is the mass density, $\cong$ is the elasticplastic tangent modulus depending on the material properties $(\mathrm{E}, \nu)$, the stress and the deformation history. The tensor $\underline{\mathbb{d}}$ denotes the rate of deformation tensor, $\underline{\mathbb{K}}$ the temperature dependence of the material properties including the thermal expansion, $\mathrm{T}$ the absolute temperature and $\phi^{\mathrm{t}}$ represents the dissipation related to the time dependent material behaviour.

For isotropic materials, the respective terms read as follows:

$$
\begin{gathered}
\underline{\mathbb{K}}=-(1-h) \underline{Y}^{*}+\frac{1}{G} \frac{d G}{d t} \underline{\sigma}+\frac{3 \rho}{\rho_{0}} C_{b} \alpha \underline{I} \\
\underline{\mathbb{C}}=\underline{\underline{L}}^{*}-(1-h) \underline{\underline{Y}} \\
\Phi^{t}=\frac{3 \xi}{2 \sigma^{2}} \underline{\underline{\mathbb{C}}}: \underline{\underline{s}}
\end{gathered}
$$

where $\mathrm{h}$ is the hardening modulus, $\underline{\mathrm{Y}}^{*}$ a tensor related to thermal exertion of yielding, $\mathrm{G}$ the shear modulus, $\mathrm{C}_{\mathrm{b}}$ the bulkmodulus, $\alpha$ the coefficient of thermal expansion, $\underline{\underline{L}}^{*}$ the elasticity deformation tensor for large deformations, $\underline{\underline{Y}}$ the yield tensor (dependent on $\underline{L}^{*}$, the yield potential and $\underline{\sigma}$ ), $\xi$ $=\xi\left(\sigma \rho_{0} / \rho, \mathrm{T}\right)$ represents the energy dissipation function, $\sigma$ the equivalent deviatoric stress and $\underline{s}$ the deviatoric stress tensor $(\underline{s}=\underline{\sigma}-1 / 3 \underline{I}$ tr $\underline{\sigma})$. In the case that the elastic part of the deformation is small (which is representative for extrusion), the yield tensor reads (for more details see [1],[2],[11]):

$$
\underline{\underline{Y}}=\frac{3 G \underline{\underline{\underline{s}} \underline{\underline{S}}}}{\sigma^{2}}
$$

\section{EXAMPLES OF FORMING PROCESSES}

In this section two examples are given of the calculation of forming processes. The calculations are performed with Dieka, a Finite Element Programm developed by Huétink et al., in which the aforementioned descriptions are built in. Particular results will be shown.

First, the deformation of an extrusion die during extrusion is considered. As an example a die for hollow profiles (with one or more holes enclosed in the cross-section of the profile) is treated since 
its exact behaviour during extrusion is not known yet. One important property of hollow dies is the fact that the inner surface is formed by a core which is suspended by three or more legs in the die. So, the aluminum flow is split by the legs, and after the legs the flow has to weld together again (in so-called welding chambers, see Fig.1). It can be understood that the friction forces on the legs form an important part of the total loading of the die. In the underlying calculation of a die for the extrusion of a simple circular tube, the friction forces are calculated in a two-dimensional space. This can be assumed to be a good approximation because motion of the flow perpendicular to the extrusion direction between two legs is negligible. The finite element model used is shown in Fig. 1, being an eccentric cross section of the die (therefore, the modelled outlet is only present to enable any flow; in the real cross-section no outlet is present in this cross-section). Only one half of the model has to be considered because of symmetry. The dotted lines represent the area with so-called contact elements, modelling the contact and (Coulomb) friction. Plane strain elements are used. The extrusion ratio is 110 which hinders large prescribed displacements at the right side of the model (because of the stability criterion, eq.(5)). Some results are given in Figs. 2 and 3.

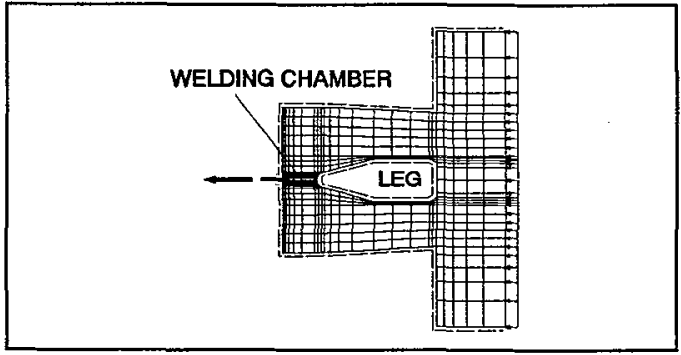

Fig. 1 Mesh for 2D calculation

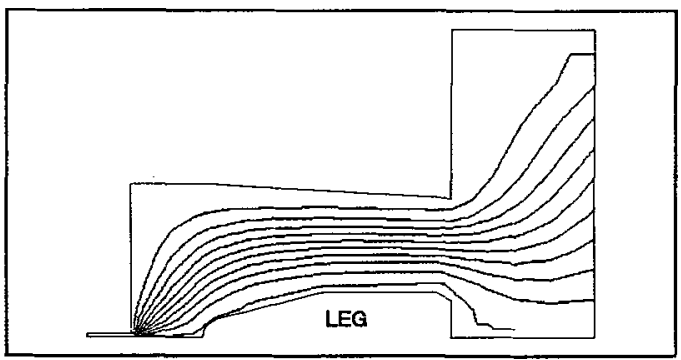

Fig. 3 Streamlines around leg

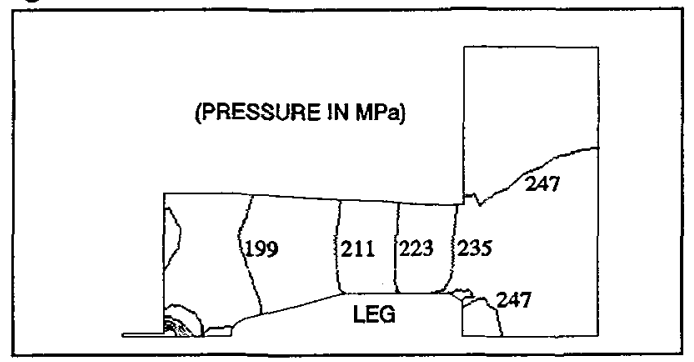

Fig. 2 Pressure distribution

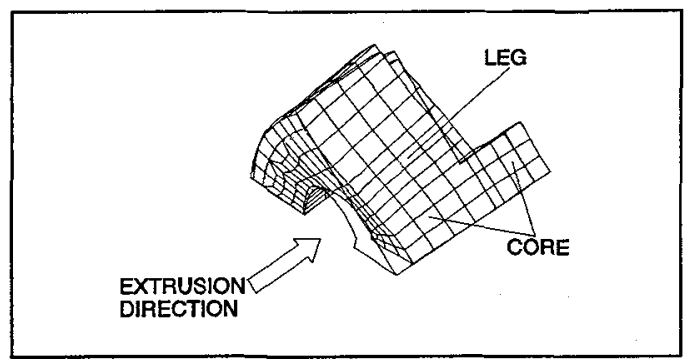

Fig. 4 3D mesh of a part of the extrusion die

The straight pressure profile indicates a flow that is mainly governed by friction forces at the walls (Poisseuille distribution). The largest pressure drop is found, as expected, near the outlet. The small pressure drop in the (diverging!) inside canal is also well predicted. The streamlines (Fig. 3) perfectly show the expected dead-metal zones. If it is assumed that the friction between the die and the aluminum is of the same magnitude as the internal friction force of the aluminum, so, there is no necessity for rounding the channels in the die. In a separate calculation a three-dimensional model of a die (one eighth, because of symmetry, see Fig.4) is considered. The loading caused by friction as well as the pressure on the core result from the two-dimensional calculation. The deflection of the core is calculated to be a few tenths of a millimetre (see Fig. 4; the plotted deflections are exaggerated!). Because this deflection directly influences the formed profile it is very important for the design of the die. No plastic deformation of the die is predicted by these rather coarse calculations. In the near future, the effects of temperature, creep and rate dependent material behaviour will be considered.

As a second example the simulation of metal sheet rolling is shown (Fig. 5). Again, only one half of the set-up is modelled because of symmetry. The angular velocity of the rolls is prescribed. The 
contact between the rolls and the sheet (including friction) is described by contact elements. The residual stresses in the outflow are shown, which are important for sheet products. A result of the stresses in two layers is shown in Fig. 6, respectively at the surface and at the centre line of the sheet. Results for the accelerated method are shown in the same figure as well. Since almost the same results are accomplished in almost a factor 3.1 less increments (2294 steps against 743 steps), the method can be said to be successful which is interesting for control purposes. Analogous pictures can be shown for the plastic strain distribution calculated within even fewer increments.

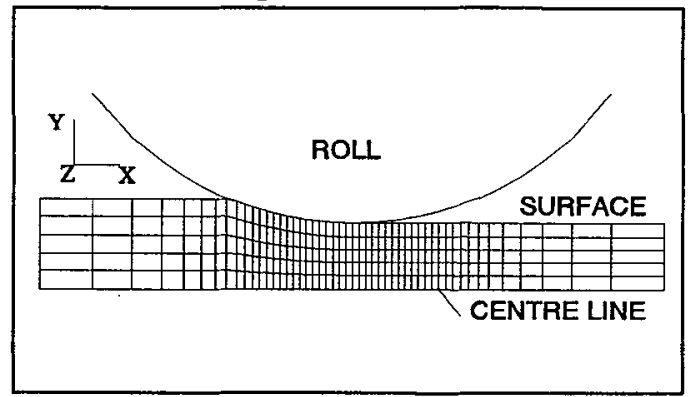

Fig. 5 Mesh for rolling (thickness exaggerated)

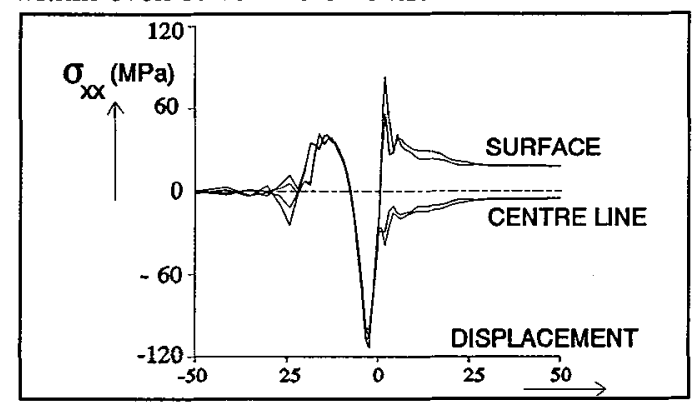

Fig. 6 Longitudinal stresses in two layers

\section{CONCLUSIONS}

The Finite Element code Dieka has been used successfully to simulate (stationary) forming processes. For aluminium extrusion, a combination of a two dimensional calculation of the flow to predict the loading forces of the flow on the die and a three dimensional calculation of the deformation of the die seems to yield reasonable results. Interesting design and control properties of the extrusion process can be inquired, such as: plastic deformation of the die, friction forces and the occurrence of deadmetal zones. A method to accelerate (transient) calculations of forming processes by local increment magnification gives good results: the calculations can be speeded up by a factor 4 to 10 (for stresses and strains, respectively) whereas the results are unaffected. The acceleration method is successfully applicated to e.g. the simulation of metal sheet rolling.

7. ACKNOWLEDGEMENTS: AMAG/BOAL B.V. and the Hoogovens Group are gratefully acknowledged for the cooperation and financial support. The authors want to thank mr. R.L. van Dijk for carrying out the extrusion calculations.

\section{REFERENCES}

[1] Hibbitt, H.B., Marcal,P.V. and Rice, J.R., 'A finite element formulation for problems of large strain and large displacement', Int. J. Sol. Structures, Vol.6'(1970), pp. 1069-1086.

[2] McMeeking, R.M., Rice, J.R., 'Finite element formulation for problems of large elastic-plastic deformation', Int.J. of Sol. Structures, Vol.11 (1975), pp. 601-616.

[3] Gelten, C.J.M. and Konter, A.W.A., Application of mesh-rezoning in the updated Lagrangian method to metal forming analysis', In: J.F.T. Pittman et al. (eds), Num. meth. in indust. forming processes, Peneridge Press, 1982, pp. 511-521.

[4] Hughes, T.J.R., Liu, W.K. and Zimmermann, T.K.,' Lagrangian-Eulerian finite element formulation for incompressible viscous flow', Comp.Meth.Appl.Mech.Eng., Vol.29 (1981), pp.329-349.

[5] Donea, J., 'Arbitrary Lagrangian-Eulerian finite element method', Comp.meth. for transient analysis, In: T. Belytschko and Hughes (eds), Comp.Meth. in Mech. , North Holland, Vol.1.

[6] Liu, W.K., Belytschko, T. and Chang, H., 'An Arbitrary Lagrangian-Eulerian finite element method for path dependent materials', Comp.Meth.Appl.Mech.Eng., Vol.58(1986),pp 227-245.

[7] Huétink, J., 'Analysis of metal forming proc. based on a comb. Euler-Lagrangian FE formulation', In: J.F.T. Pittman et al. (eds), Num, meth. in indust. forming processes, 1982, Peneridge press, pp. 501-509.

[8] Hinton, E. and Gampbell, J.S., 'Local and global smoothing of discontinuous finite element functions using least square method', Int.J.Num.Meth. Eng., Vol.8 (1974), pp. 461-480.

[9] Huétink, J. and Helm, P.N. van der, 'On Euler-Lagrange finite element formulation in forming and fluid problems', In: Chenot J.-L. e.a., 'Num.Meth. in Industr. Forming Proc.', (1992), Balkema Rotterdam, pp. 45-54.

[10] Meijers, E.M., 'Efficiency Enhancement of a mixed Eulerian-Lagrangian FE-method for the simulation of steadystate rolling processes', Mech.Eng. (1992), University of Twente.

[11] Huétink, J., 'On' the simulation of thermomechanical forming processes', (1986), Dissertation, University of Twente, The Netherlands. 